\title{
Atuação da fisioterapia nos cuidados paliativos em pacientes oncológicos: Uma revisão integrativa
}

Physiotherapy performance in palliative care in oncological patients: An integrative review

Desempeño de fisioterapia en cuidados paliativos en pacientes oncológicos: Una revisión integradora

Randresson Jadson Ferreira Silva ORCID: https://orcid.org/0000-0001-5454-9080 Centro Universitário de Ciências e Tecnologia do Maranhão, Brasil E-mail: randresson.cx@gmail.com

Kaio Germano Sousa da Silva

ORCID: https://orcid.org/0000-0003-4236-6230 Centro Universitário de Ciências e Tecnologia do Maranhão, Brasil
E-mail: kaiogsds@hotmail.com

Letícia Aparecida Santos Silva ORCID: https://orcid.org/0000-0002-8000-7071 Centro Universitário de Ciências e Tecnologia do Maranhão, Brasil E-mail: leticia.odonto01@ outlook.com

Kameny Santos Franco

ORCID: https://orcid.org/0000-0001-6056-3663

Centro Universitário Santo Agostinho, Brasil E-mail: kamenyfranco@gmail.com

Chrisllayne Oliveira da Silva

ORCID: https://orcid.org/0000-0002-0844-0268 Centro Universitário de Ciências e Tecnologia do Maranhão, Brasil E-mail: chris-layne10@ hotmail.com

Pammela Weryka da Silva Santos ORCID: https://orcid.org/0000-0002-9931-0951

Universidade Estadual do Maranhão, Brasil

E-mail: pammela_weryka@hotmail.com

Pedro Henrique Medeiros de Andrade

ORCID: https://orcid.org/0000-0003-1819-9540

Centro Universitário de Ciências e Tecnologia do Maranhão, Brasil E-mail: pedro.medeiroscx@gmail.com

Rita de Cássia Helen Alves de Sousa Quadros

ORCID: https://orcid.org/0000-0003-2972-2480

Centro Universitário de Ciências e Tecnologia do Maranhão, Brasil E-mail: rhsousaa@gmail.com

Adelaide da Paz Correia

ORCID: https://orcid.org/0000-0002-6405-568X

Centro Universitário de Ciências e Tecnologia do Maranhão, Brasil

E-mail: adelaide_paz@hotmail.com

Fernando Magno Freitas de Amorim

ORCID: https://orcid.org/0000-0002-5396-0561

Centro Universitário de Ciências e Tecnologia do Maranhão, Brasil

E-mail: fernandomagnocx@ hotmail.com

Francisco de Assis Viana dos Santos

ORCID: https://orcid.org/0000-0003-3047-260X Universidade Federal do Piauí, Brasil E-mail: assissantosf9@gmail.com

Raydelane Grailea Silva Pinto

ORCID: https://orcid.org/0000-0001-8657-6462 Universidade Federal do Piauí, Brasil

E-mail: raydelane.silva@gmail.com

Juliana Rocha de Sousa

ORCID: https://orcid.org/0000-0002-8533-5998

Centro Universitário de Ciências e Tecnologia do Maranhão, Brasil E-mail: jrochafisio2@gmail.com 


\begin{abstract}
Resumo
O fisioterapeuta em oncologia exerce função quanto prevenção, tratamento e na paliação das disfunções em qualquer momento do tratamento do câncer, como no diagnóstico, pré, peri e pós-cirúrgico, nos efeitos dos tratamentos como quimioterapia, radioterapia, hormonioterapia, imunoterapia ou mesmo no cuidado paliativo exclusivo. Esse estudo teve como objetivo analisar as produções cientificas referente a atuação da fisioterapia nos cuidados paliativos em pacientes oncológicas. Trata-se de uma pesquisa bibliográfica do tipo revisão integrativa, com a seguinte questão norteadora: Qual a importância da atuação do fisioterapeuta nos cuidados paliativos em pacientes oncológicos? Foram utilizados descritores e as bases de dados consultadas foram: Literatura Latino-Americana e do Caribe em Ciências da Saúde (LILACS); Scientific Electronic Library Online (SCIELO) e Medical Literature Analysis and Retrieval System Online (Medline). A amostra final de estudos selecionados nas bases de dados foi composta de 12 artigos científicos, levando em consideração os critérios de inclusão e exclusão estabelecidos neste estudo, na qual foram extraídos diretamente das bases de dados, e avaliados quanto a elegibilidade para responder à questão norteadora proposta na pesquisa. O estudo buscou através da literatura cientifica compreender a atuação do fisioterapeuta nos cuidados paliativos. Esse profissional tem um papel fundamental como membro da equipe multidisciplinar e no acompanhamento dos pacientes oncológicos, pois a fisioterapia possui uma extensa área de conhecimentos que podem ser utilizados e colocados em prática para o desenvolvimento de planos de cuidados na assistência prestada aos pacientes oncológicos.
\end{abstract}

Palavras-chave: Cuidados paliativos; Fisioterapia; Humanização da assistência.

\begin{abstract}
The oncology physiotherapist plays a role in preventing, treating and palliating dysfunctions at any time during cancer treatment, such as in diagnosis, pre, peri and post-surgery, in the effects of treatments such as chemotherapy, radiotherapy, hormone therapy, immunotherapy or even in exclusive palliative care. This study aimed to analyze the scientific productions regarding the performance of physiotherapy in palliative care in cancer patients. This is an integrative review bibliographic search, with the following guiding question: What is the importance of the physiotherapist's performance in palliative care in cancer patients? Descriptors were used and the databases consulted were: Latin American and Caribbean Literature on Health Sciences (LILACS); Scientific Electronic Library Online (SCIELO) and Medical Literature Analysis and Retrieval System Online (Medline). The final sample of studies selected from the databases was composed of 12 scientific articles, taking into account the inclusion and exclusion criteria established in this study, in which they were extracted directly from the databases, and assessed for eligibility to answer the guiding question. proposed in the research. The study sought through the scientific literature to understand the role of the physiotherapist in palliative care. This professional has a fundamental role as a member of the multidisciplinary team and in the monitoring of cancer patients, as physiotherapy has an extensive area of knowledge that can be used and put into practice for the development of care plans in the care provided to cancer patients.
\end{abstract}

Keywords: Palliative care; Physiotherapy; Humanization of assistance.

\title{
Resumen
}

El fisioterapeuta oncológico juega un papel en la prevención, el tratamiento y la paliación de disfunciones en cualquier momento durante el tratamiento del cáncer, como en el diagnóstico, pre, peri y poscirugía, en los efectos de tratamientos como quimioterapia, radioterapia, hormonoterapia, inmunoterapia o incluso en cuidados paliativos exclusivos. Este estudio tuvo como objetivo analizar las producciones científicas sobre el desempeño de la fisioterapia en cuidados paliativos en pacientes con cáncer. Se trata de una búsqueda bibliográfica de revisión integradora, con la siguiente pregunta orientadora: ¿Cuál es la importancia del desempeño del fisioterapeuta en cuidados paliativos en pacientes oncológicos? Se utilizaron descriptores y las bases de datos consultadas fueron: Literatura Latinoamericana y del Caribe en Ciencias de la Salud (LILACS); Biblioteca electrónica científica en línea (SCIELO) y Sistema de análisis y recuperación de literatura médica en línea (Medline). La muestra final de estudios seleccionados de las bases de datos estuvo compuesta por 12 artículos científicos, teniendo en cuenta los criterios de inclusión y exclusión establecidos en este estudio, en los que se extrajeron directamente de las bases de datos, y se evaluó su elegibilidad para responder a la pregunta orientadora. en la investigación. El estudio buscó a través de la literatura científica comprender el papel del fisioterapeuta en los cuidados paliativos. Este profesional tiene un papel fundamental como miembro del equipo multidisciplinar y en el seguimiento de los pacientes oncológicos, ya que la fisioterapia cuenta con una amplia área de conocimiento que puede ser utilizada y puesta en práctica para el desarrollo de planes asistenciales en la atención que se brinda a pacientes con cáncer.

Palabras clave: Cuidados paliativos; Fisioterapia; Humanización de la asistencia.

\section{Introdução}

A palavra "Câncer" deriva do grego "Karkinos", que significa caranguejo, utilizada pela primeira vez pelo pai da medicina Hipócrates, onde câncer não é uma doença nova, havendo indícios sobre a sua existência em múmias egípcias com 
mais de 3 mil anos (Instituto Nacional de Câncer [INCA], 2019). O câncer é um termo que define um conjunto de várias doenças decorrentes do crescimento desorganizado das células malignas. Essas células invadem outros tecidos e órgãos, podendo se espalhar para outras partes do corpo através da corrente sanguínea ou linfática, gerando metástase (Cazzaniga \& Bonanni, 2018).

Segundo a Organização Mundial da Saúde (OMS), o câncer é uma das principais causas de morte no mundo, respondendo por 8,8 milhões de mortes em 2015. As causas mais comuns de morte por câncer são os cânceres de pulmão (1,69 milhões de mortes), fígado (788.000 mortes), colorretal (774.000 mortes), estômago (754.000 mortes) e mama (571.000 mortes) (World Health Organization, 2014).

O fisioterapeuta em oncologia exerce função quanto prevenção, tratamento e na paliação das disfunções em qualquer momento do tratamento do câncer, como no diagnóstico, pré, peri e pós-cirúrgico, nos efeitos dos tratamentos como quimioterapia, radioterapia, hormonioterapia, imunoterapia ou mesmo no cuidado paliativo exclusivo (Matheus, Silva, \& Figueiredo, 2018).

A fisioterapia também disponibiliza diversas técnicas que podem ser utilizadas em pacientes oncológicos, como a eletroterapia, cinesioterapia, termoterapia, crioterapia e entre outras, essas técnicas podem ser aplicadas em conjunto com outros profissionais visando o bem estar e uma assistência efetiva (Nascimento, Marinho, \& Costa, 2017).

Nesse contexto, o fisioterapeuta atua nos cuidados paliativos principalmente de maneira a minimizar o sofrimento, a fim de evitar maiores complicação. Onde a avaliação fisioterapêutica é importante para diminuir o risco de mais complicações a partir de uma grande variedade de técnicas, além de contribuir com o aspecto psicossocial, restaurando o senso de dignidade, para nortear a intervenção e a evolução clínica do paciente desde o momento do prognóstico até o processo de morte (Freitas, Gonçalves \& Morais, 2016).

Utilizou-se como questão norteadora do estudo: Qual a atuação da fisioterapia nos cuidados paliativos de pacientes oncológicos? Para tal tendo como objetivo geral, analisar as produções cientificas nacionais e internacionais referente a atuação da fisioterapia nos cuidados paliativos em pacientes oncológicas, e especificamente determinar as principais condutas fisioterapêuticas no tratamento oncológico; identificar as principais complicações decorrentes do tratamento oncológico; e apontar a importância da atuação fisioterapêutica no alívio dos sintomas e melhorar a qualidade de vida de pacientes oncológicos.

\section{Metodologia}

Trata-se de uma pesquisa bibliográfica do tipo revisão integrativa com abordagem qualitativa (Sousa et al., 2017; (Marconi \& Lakatos, 2003) este tipo de estudo constitui uma análise extensa e descritiva da literatura, e através dela pode-se discutir os métodos e resultados das pesquisas e também refletir sobre pesquisas futuras. Este método permite que o pesquisador possa ter compreensão e aprofundamento da temática escolhida por meio de fontes secundárias e confiáveis de estudos publicados.

Desta forma estudos de cunho bibliográficos fornecem uma base para a produção de novos estudos, além disso é uma metodologia muito utilizada para anteceder outros estudos, pois permite o aprofundamento do tema e avaliar todas as informações já existentes no assunto, proporcionado uma nova visão acerca da temática e indagações para estudos futuros (Carvalho, Duarte, Menezes, \& Souza, 2019).

A partir da temática "Atuação Da Fisioterapia Nos Cuidados Paliativos Em Pacientes Oncológicos: uma revisão integrativa", elaborou-se a seguinte questão norteadora deste estudo: Qual a importância da atuação do fisioterapeuta nos cuidados paliativos em pacientes oncológicos? 
Por meio da definição da questão norteadora foi realizado a busca de estudos nas bases de dados, utilizando-se os descritores: cuidados paliativos, fisioterapia, oncologia e Humanização da Assistência. As bases de dados consultadas foram: Literatura Latino-Americana e do Caribe em Ciências da Saúde (LILACS); Scientific Electronic Library Online (SCIELO) e Medical Literature Analysis and Retrieval System Online (Medline).

Como critérios de inclusão, foram selecionados estudos em português, inglês e espanhol, que fossem textos completos e que abordassem a temática em questão, com o recorte temporal entre os anos 2009 e 2019. Os critérios de exclusão foram resumos, textos incompletos, fora do recorte temporal estabelecido e que não estivessem condizentes com a pergunta norteadora.

Os descritores foram combinados e aplicados as bases de dados, na qual foi utilizado o booleano "AND". Ao todo foram encontrados 180 artigos nas bases de dados, na LILACS resultou em 60 artigos, após a aplicação dos critérios de inclusão, 30 artigos estavam disponíveis, em seguida foi realizado a leitura de títulos e resumos resultando em 12 estudos, após a leitura dos textos completos somente três (03) foram incluídos.

Na MEDLINE resultou em 59 artigos, após a aplicação dos critérios de inclusão resultou em 30 artigos disponíveis, em seguida foi realizado a leitura de títulos e resumos resultando em 17 estudos, após a leitura dos textos completos restaram oito (08) estudos, sendo que apenas três (03) atendiam a questão norteadora e foram incluídos no estudo.

$\mathrm{Na}$ base de dados SCIELO foram encontrados 61 artigos no total, com a aplicação dos critérios de inclusão resultou em 18 artigos, após a leitura de título e resumos, somente sete (07) estudos foram selecionados, depois da leitura na íntegra, apenas seis (06) foram incluídos na amostra final, como pode ser observado na Figura 1.

Figura 1. Distribuição da quantidade de artigos selecionados nas bases LILACS, MEDLINE e SCIELO. Caxias, MA, Brasil, 2019. N=12.

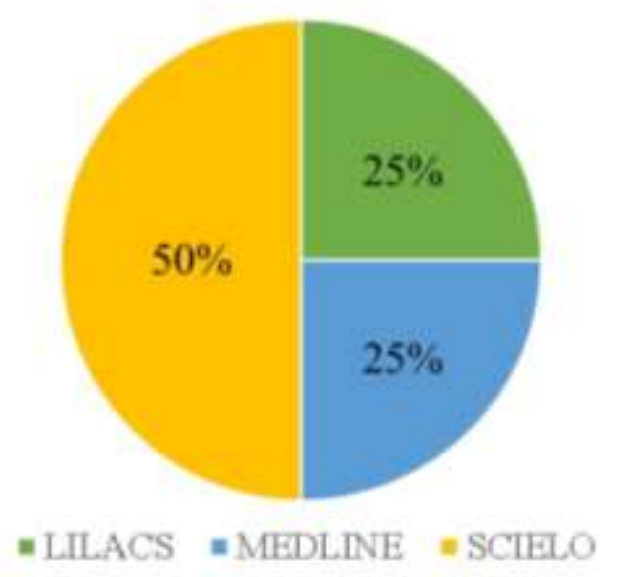

Fonte: Base de dados (2019).

Para análise e interpretação dos resultados, as informações coletadas nos estudos científicos foram analisadas e ordenadas de forma descritiva em quadros contendo as informações relevantes acerca dos estudos, para facilitar a compreensão sobre os estudos incluídos. Esta pesquisa levou em consideração os aspectos éticos das pesquisas em relação as citações dos estudos, respeitando as ideias autorais, os conceitos e as definições presentes nos artigos incluídos nesta revisão. 


\section{Resultados e Discussão}

A amostra final de estudos selecionados nas bases de dados foi composta de 12 artigos científicos, levando em consideração os critérios de inclusão e exclusão estabelecidos neste estudo, na qual foram extraídos diretamente das bases de dados, e avaliados quanto a elegibilidade para responder à questão norteadora proposta no estudo conforme o Quadro 1.

Quadro 1. Classificação dos estudos quanto ao Autor/ano, título e base de dados. Caxias, MA Brasil, 2019.

\begin{tabular}{|c|c|c|}
\hline AUTOR/ANO & TITULO & BASE DE DADOS \\
\hline Azevedo (2015) & Cuidados paliativos: Tratamento fisioterapêutico em pacientes com câncer. & LILACS \\
\hline Batista e Guarnieri (2019) & $\begin{array}{l}\text { A importância do atendimento fisioterapêutico humanizado no paciente } \\
\text { oncológico: uma revisão literária. }\end{array}$ & LILACS \\
\hline Cunha e Gardenghi (2019) & $\begin{array}{l}\text { A fisioterapia nos cuidados paliativos a pacientes com câncer - uma } \\
\text { revisão baseada em evidências }\end{array}$ & LILACS \\
\hline Bernardo (2012) & $\begin{array}{l}\text { Pacientes oncológicos em cuidados paliativos: Correlação entre qualidade } \\
\text { de vida e funcionalidade. }\end{array}$ & SCIELO \\
\hline Costa (2017) & $\begin{array}{l}\text { Cuidados paliativos ao paciente com câncer: Atuação do fisioterapeuta no } \\
\text { âmbito hospitalar. }\end{array}$ & MEDLINE \\
\hline $\begin{array}{l}\text { Müller, Scortegagna e } \\
\text { Moussalle (2011) }\end{array}$ & $\begin{array}{l}\text { Paciente Oncológico em Fase Terminal: Percepção e Abordagem do } \\
\text { Fisioterapeuta }\end{array}$ & MEDLINE \\
\hline Burgos (2017) & Fisioterapia Paliativa Aplicada ao Paciente Oncológico Terminal & MEDLINE \\
\hline $\begin{array}{l}\text { Baldini, Salles e Santana } \\
\text { (2010) }\end{array}$ & $\begin{array}{l}\text { A atuação do fisioterapeuta no controle da dor oncológica: Uma revisão de } \\
\text { literatura. }\end{array}$ & SCIELO \\
\hline Duarte (2018) & $\begin{array}{l}\text { Atuação do fisioterapeuta em pacientes oncológicos em cuidados paliativos } \\
\text { em um hospital filantrópico da cidade de Maceió. }\end{array}$ & SCIELO \\
\hline $\begin{array}{l}\text { Goés, Munduruca, Ferreira } \\
\text { e Passos (2016) }\end{array}$ & $\begin{array}{l}\text { Atuação do fisioterapeuta nos cuidados paliativos em pacientes } \\
\text { oncológicos adultos hospitalizados: Revisão de literatura }\end{array}$ & SCIELO \\
\hline $\begin{array}{l}\text { Oliveira, Bombarda e } \\
\text { Moriguchi (2019) }\end{array}$ & $\begin{array}{l}\text { Fisioterapia em cuidados paliativos no contexto da atenção primária à } \\
\text { saúde: ensaio teórico. }\end{array}$ & SCIELO \\
\hline Silva (2014) & Recursos fisioterapêuticos no tratamento da dor oncológica. & SCIELO \\
\hline
\end{tabular}

Fonte: Base de dados (2019).

As buscas realizadas com base na pergunta norteadora do estudo indicaram uma maior quantidade de publicações na base SCIELO, com o ano de 2017 e 2019 tendo mais estudos encontrados. Os estudos foram avaliados, discutidos e extraídos as informações principais dos mesmos, como demonstra o Quadro 2.

Quadro 2. Estudos de acordo com os autores, título e principais resultados. Caxias, MA, Brasil, 2019.

\begin{tabular}{|c|l|l|}
\hline AUTOR/ANO & \multicolumn{1}{|c|}{ TITULO Raliativos: Tratamento } & \multicolumn{1}{|c|}{ RESULTADO PRINCIPAL } \\
\hline Azevedo (2015) & $\begin{array}{l}\text { Cuidados possível identificar o papel do fisioterapeuta na equipe } \\
\text { fisioterapêutico em pacientes com } \\
\text { câncer. }\end{array}$ & $\begin{array}{l}\text { Foi } \\
\text { multidisciplinar que se dedica aos cuidados paliativos por meio de } \\
\text { técnicas fisioterapêuticas, vem demonstrando que os mesmos, podem } \\
\text { proporcionar uma melhor qualidade de vida para os indivíduos com } \\
\text { doenças crônicas e ameaçadoras à vida e consequentemente aos seus } \\
\text { familiares. }\end{array}$ \\
\hline $\begin{array}{c}\text { Batista e Guarnieri } \\
\text { (2019) }\end{array}$ & $\begin{array}{l}\text { A importância do atendimento } \\
\text { fisioterapêtico humanizado no } \\
\text { paciente oncológico: uma revisão } \\
\text { literária. }\end{array}$ & $\begin{array}{l}\text { Observa-se que é de vital a importância do atendimento fisioterápico } \\
\text { humanizado no paciente oncológico. Conclui-se também que tornar } \\
\text { uma assistência humanizada é revelar os valores que constituem o ser } \\
\text { humano como pessoa de forma abrangente e completa, o que gera, em } \\
\text { consequência melhora na qualidade de vida do paciente oncológico, } \\
\text { uma vez que apoio e força propiciam um diferencial no tratamento }\end{array}$ \\
\hline Bernardo (2012) & $\begin{array}{l}\text { Pacientes oncológicos em cuidados } \\
\text { paliativos: Correlação entre qualidade } \\
\text { de vida e funcionalidade. }\end{array}$ & $\begin{array}{l}\text { A maioria dos pacientes investigados apresentaram baixo escore de } \\
\text { qualidade de vida e de funcionalidade, e apesar da pequena amostra } \\
\text { deste estudo (n=15), obtiveram-se resultados satisfatórios. Salienta-se a } \\
\text { necessidade da realização de novas pesquisas com relação à QV e a } \\
\text { funcionalidade dos pacientes oncológicos em cuidados paliativos, se } \\
\text { possível, com um número maior de sujeitos. }\end{array}$ \\
\hline
\end{tabular}




\begin{tabular}{|c|c|c|}
\hline Costa (2017) & $\begin{array}{l}\text { Cuidados paliativos ao paciente com } \\
\text { câncer: Atuação do fisioterapeuta no } \\
\text { âmbito hospitalar. }\end{array}$ & $\begin{array}{l}\text { Nota-se o grande potencial de atuação da fisioterapia nos cuidados do } \\
\text { paciente oncológico sem possibilidade de cura. Entretanto a escassez de } \\
\text { estudos científicos sobre o papel e estratégias acerca da atuação desses } \\
\text { profissionais ainda é escassa. }\end{array}$ \\
\hline $\begin{array}{l}\text { Cunha e Gardenghi } \\
\text { (2019) }\end{array}$ & $\begin{array}{l}\text { A fisioterapia nos cuidados paliativos a } \\
\text { pacientes com câncer }- \text { uma revisão } \\
\text { baseada } \\
\text { em }\end{array}$ & $\begin{array}{l}\text { Compreende-se que a fisioterapia tem um papel relevante e } \\
\text { imprescindível nos cuidados paliativos, entretanto necessita-se de uma } \\
\text { maior oferta e implementação deste tratamento nos serviços de saúde. }\end{array}$ \\
\hline $\begin{array}{c}\text { Müller, } \\
\text { Scortegagna e } \\
\text { Moussalle (2011) }\end{array}$ & $\begin{array}{l}\text { Paciente Oncológico em Fase Terminal: } \\
\text { Percepção e Abordagem do } \\
\text { Fisioterapeuta }\end{array}$ & $\begin{array}{l}\text { Constatou-se através da análise das entrevistas que a relação } \\
\text { estabelecida entre o fisioterapeuta e o paciente oncológico em fase } \\
\text { terminal, por mais difícil que seja lidar com esta situação, é de extrema } \\
\text { importância para ambos e é evidenciada neste estudo como uma relação } \\
\text { de amizade, afetividade e compreensão, necessária para a terapêutica } \\
\text { do paciente, afim deste se sentir importante, querido e não abandonado. } \\
\text { Este estudo revela que o papel do fisioterapeuta diante do paciente } \\
\text { oncológico terminal vai muito além do seu trabalho técnico e trata } \\
\text { muito mais do que a condição física do seu paciente. }\end{array}$ \\
\hline Burgos (2017) & $\begin{array}{l}\text { Fisioterapia Paliativa Aplicada ao } \\
\text { Paciente Oncológico Terminal }\end{array}$ & $\begin{array}{l}\text { Os resultados obtidos apontam para relevância dos cuidados paliativos } \\
\text { na assistência ao paciente oncológico para a necessidade de novas } \\
\text { pesquisas cientificas relativa ao tema. }\end{array}$ \\
\hline $\begin{array}{l}\text { Baldini, Salles e } \\
\text { Santana (2010) }\end{array}$ & $\begin{array}{l}\text { A atuação do fisioterapeuta no controle } \\
\text { da dor oncológica: Uma revisão de } \\
\text { literatura. }\end{array}$ & $\begin{array}{l}\text { Os resultados demonstraram uma falta de fundamentação científica } \\
\text { para a utilização dos recursos fisioterapêuticos no tratamento da dor } \\
\text { oncológica, ao passo que ratificaram que os estudos desenvolvidos, até } \\
\text { agora, não oferecem evidências suficientes para recomendar ou rejeitar } \\
\text { a utilização dos recursos citados para o controle da dor do paciente com } \\
\text { câncer. }\end{array}$ \\
\hline Duarte (2018) & $\begin{array}{l}\text { Atuação do fisioterapeuta em pacientes } \\
\text { oncológicos em cuidados paliativos em } \\
\text { um hospital filantrópico da cidade de } \\
\text { Maceió. }\end{array}$ & $\begin{array}{l}\text { Foi possível observar que o fisioterapeuta detém métodos e recursos } \\
\text { exclusivos de sua profissão que são imensamente úteis nos cuidados } \\
\text { paliativos, e sua atuação corrobora com o tratamento multiprofissional } \\
\text { e integrado necessário para o atendimento de pacientes com câncer. }\end{array}$ \\
\hline $\begin{array}{l}\text { Goés, Munduruca, } \\
\text { Ferreira e Passos } \\
\text { (2016) }\end{array}$ & $\begin{array}{l}\text { Atuação do fisioterapeuta nos cuidados } \\
\text { paliativos em pacientes oncológicos } \\
\text { adultos hospitalizados: revisão de } \\
\text { literatura. }\end{array}$ & $\begin{array}{l}\text { Devido ao potencial benefício da inserção da fisioterapia nos cuidados } \\
\text { paliativos, é necessário difundir aos fisioterapeutas a discussão de } \\
\text { temas relacionados à humanização, morte e cuidados paliativos e a } \\
\text { realização de maiores investigações para otimizar a atuação deste } \\
\text { profissional nos processos oncológicos. }\end{array}$ \\
\hline $\begin{array}{l}\text { Oliveira, Bombarda } \\
\text { e Moriguchi (2019) }\end{array}$ & $\begin{array}{l}\text { Fisioterapia em cuidados paliativos no } \\
\text { contexto da atenção primária à saúde: } \\
\text { ensaio teórico }\end{array}$ & $\begin{array}{l}\text { As contribuições do fisioterapeuta nos cuidados paliativos no contexto } \\
\text { da atenção primária ainda necessitam de estruturação e qualificação. É } \\
\text { necessário compreender a realidade de trabalho vivenciada por esse } \\
\text { profissional nesse nível de atenção, pois a Fisioterapia tenha um campo } \\
\text { abrangente de técnicas para acrescer nos cuidados paliativos ofertados, } \\
\text { são necessários mais estudos neste campo da fisioterapia que aborde as } \\
\text { condutas e a efetividades das mesmas na prestação de cuidados. }\end{array}$ \\
\hline Silva (2014) & $\begin{array}{l}\text { Recursos fisioterapêuticos } \\
\text { tratamento da dor oncológica. }\end{array}$ & $\begin{array}{l}\text { Os cuidados paliativos tem como objetivo suprir as necessidades } \\
\text { específicas de pacientes sem possibilidades terapêuticas. Sua inclusão } \\
\text { no atendimento de pacientes } \\
\text { oncológicos é de extrema importância, sendo uma necessidade } \\
\text { crescente nos hospitais e instituições de saúde. O } \\
\text { fisioterapeuta detém métodos e recursos exclusivos de sua profissão } \\
\text { que são imensamente úteis nos cuidados paliativos. }\end{array}$ \\
\hline
\end{tabular}

Fonte: Dados da pesquisa (2019).

$\mathrm{Na}$ busca de compreender da resolutividade a gestão social no contexto humanitário e no tratamento humanizado em pacientes oncológicos, destaca-se a importância do profissional de fisioterapia em oferecer tratamento paliativo humanizado como forma de oferecer uma qualidade de vida aos pacientes e a família, que sofrem desde do diagnóstico e durante todo o tratamento (Azevedo, 2015).

Os tratamentos oferecidos pelos profissionais da fisioterapia são inúmeros, muitos destes métodos de intervenções são uteis para paciente oncológico, deste modo o profissional de fisioterapia precisa antes de tudo se adequar aos aspectos éticos e fisiológicos exigidos no tratamento individual de cada paciente (Cunha \& Gardenghi, 2019; Silva, 2014). Novas estratégias e metodologia são fundamentais para que o profissional de fisioterapia possa inovar em tecnologias eficientes, mas manter a 
comunicação com usuário e família e demais profissionais, cultivar responsavelmente a independência e a esperança deste paciente lidar com os momentos difíceis durante este processo constituem de pilares fundamentais da assistência profissional em cuidados paliativos (Goés, Munduruca, Ferreira, \& Passos, 2016).

De acordo com Burgos (2017) a inserção da fisioterapia nos cuidados paliativos é necessário e deve ter um maior compartilhamento e recrutamento de profissionais nessa área. A função do fisioterapeuta nos cuidados paliativos não acontece de forma individual, mas sim como um membro da equipe multiprofissional, que desta forma consegue ter uma visão holística do paciente e oferecer tratamento paliativo de forma humanizada e focada nas necessidades do paciente.

Consonância Duarte (2018), afirma que esse trabalho em equipe é de extrema importância, pois o paciente oncológico sofre com muitas alterações a nível físico, social e psicológico, lidar com múltiplos tratamentos, medicações, mudanças de estilo de vida, provocam muitas vulnerabilidades, o trabalho em equipe garante que em todos os níveis biopsicossociais esse paciente será atendido e escutado, minimizando os riscos e tornando os cuidados durante essa fase menos agressivos.

Nos estudos de Müller et al. (2011) é citado uma das consequências devida à patologia do câncer, que é a dor oncológica, está se caracteriza de cunho físico e emocional, e nesse contexto a importância do tratamento fisioterapêutico em diminuir o processo debilitante e ajudar esses pacientes no alívio da dor e no desenvolvimento da sua autonomia são cruciais.

A dor oncológica retrocede o quadro de saúde e contribui enormemente para a perda dos mecanismos funcionais reduzindo a independência do indivíduo. Contudo, esse processo debilitante pode ser controlado/minimizado pela interferência dos tratamentos fisioterapêuticos, que previnem a imobilidade e estimulam a autonomia do paciente, participando do tratamento com técnicas não invasivas, que aumentam o metabolismo do tecido muscular e a sua elasticidade. Entretanto, no Brasil, a fisioterapia ainda encontra dificuldade para se instalar, concretamente, como adjuvante e presença subsidiária no decorrer dos tratamentos oncológicos (Silva, 2014; Baldini, Salles \& Santana, 2010)

Para Bernardo (2012) e Batista e Guarnieri (2019) a qualidade de vida do paciente oncológico e as práticas de tratamento paliativo e a importância do profissional de fisioterapia em oferecer o tratamento humanizado, o tratamento paliativo deve estar alinhado com humanização, estratégias e implementações de saúde respeitando a individualidade de cada um, e assim ofertando cuidados de qualidade para esses pacientes.

Nos estudos de Duarte (2018) sobre a atuação fisioterapêutica em pacientes oncológico em um hospital filantrópico, observou-se que a relação profissional paciente é complexa, para o profissional ter que lidar com pacientes que não tem prognóstico de cura, pode muitas vezes representar um desafio quanto a prática de atuação, pois ao estabelecer um vínculo e uma relação de respeito com o paciente e não poder resolver o problema clínico, pode ser até mesmo frustrante, entretanto o papel dos profissionais na área de cuidados paliativos devem ser voltados em promover o bem-estar do pacientes, dignidade, e cuidado humanizado.

Deste modo Müller et al. (2011) em seu estudo sobre o paciente oncológico em fase terminal sobre o auxílio do tratamento com abordagem do profissional de fisioterapia os autores chegarem a uma seguinte correlação, constatou-se que através do analise das entrevista, que a relação estabelecida e o fisioterapeuta e o fisioterapeuta e o paciente oncológico em fase terminal, por mais difícil lidar com essa situação, e de extrema importância para ambos e evidenciou nesse estudo como uma relação de amizade, efetividade e compreensão, necessária para fisioterapia do paciente, a fim de se sentir importante, querido e não abandonado. Esse estudo revela que o papel do fisioterapeuta diante do paciente oncológico terminal vai muito além do seu trabalho técnico e muito mais do que a condição física do paciente.

O fisioterapeuta é um profissional voltado a humanização do atendimento com busca de oferecer através das suas técnicas o mínimo de dor e sequelas da doença, o mesmo trabalha diretamente com os cuidados paliativo, em que pode promover a dinamização nas estratégias de cuidados paliativos afim de proporcionar bem estar a esses indivíduos (Costa, 2017). 
O fisioterapeuta tem um papel de grande importância nos cuidados dos pacientes oncológicos sem possibilidades de cura, mas no campo de pesquisa ainda existe uma escassez de trabalho científico que comprovam os efeitos dos recursos fisioterapêuticos empregáveis, fazem-se necessário o debate sobre os temas pertinentes e a realização de maiores investigação que contribuam com o avanço do saber e o otimizarem a atuação do fisioterapeuta nos processos oncológico (Cunha \& Gardenghi, 2019).

As principais intervenções que a fisioterapia pode realizar nos cuidados do paciente com câncer são os recursos oncológico de alívio dos sintomas psicofísicos, de redução de prevenção das complicações linfáticas e de melhorar a função pulmonar (Costa, 2017). Entretanto ainda existem desafios como a falta de investimento e recursos nessa área, a falta de capacitação e despreparo profissional o que resulta em desconhecimento de informação e serviços precários (Oliveira, Bombarda \& Moriguchi, 2019).

Nota-se que a fisioterapia nos cuidados paliativo em pacientes com câncer é de extrema importância, modificar o cenário atual dos desafios no campo de atuação são necessários, todavia o papel da fisioterapia na equipe multidisciplinar que se dedica aos cuidados paliativos por meio de técnicas fisioterapêuticas demonstrando que a mesma pode proporcionar uma melhor qualidade de vida para os indivíduos com de doença crônicas é essencial em diminuir a dor oncológica, traumas e sequelas (Oliveira et al., 2019).

Em um análise detalhado dos artigos selecionado para realização deste estudo traz-se à tona a relevância do profissional de fisioterapia, a condição do trabalho humanizado, novas estratégia de tratamento de pacientes oncológico em cuidados paliativos, pois a função de oferta humanitária na melhoria dos cuidados é essencial e imprescindível nos cuidados holísticos com dignidade e respeito para os pacientes que se encontram em cuidados paliativos.

\section{Considerações Finais}

O estudo buscou através da literatura cientifica compreender a atuação do fisioterapeuta nos cuidados paliativos. Esse profissional tem um papel fundamental como membro da equipe multidisciplinar e no acompanhamento dos pacientes oncológicos, pois a fisioterapia possui uma extensa área de conhecimentos que podem ser utilizados e colocados em prática para o desenvolvimento de planos de cuidados na assistência prestada aos pacientes oncológicos.

Pacientes oncológicos estão muito vulneráveis principalmente as alterações físicas e psicológicas, durante a prestação de cuidados é necessário que o fisioterapeuta foque na comunicação e no estabelecimento de vínculos com o paciente e não somente nas técnicas, trabalhar o psicológico do paciente com a parte física é essencial para resultados positivos no tratamento, sendo a conduta profissional voltada para humanização, respeito, e confiança os pilares fundamentais para prestação de cuidados adequados.

Ainda existem muitos desafios e uma escassez de estudos voltados para atuação do fisioterapeuta em cuidados paliativos. Espera-se que o estudo contribua para a ampliação sobre a atuação do fisioterapeuta nos cuidados paliativo, permitindo a reflexão e trocas de novas possibilidades de pesquisa e intervenção., bem como estimular os profissionais atuantes de fisioterapia que buscam apreender sobre a prática no contexto de cuidados paliativos bem como relacionar suas vivências no trabalho com as transformações que vêm ocorrendo neste âmbito, estas afetam diretamente na vida de seus pacientes e as metodologias aplicadas.

Por fim, o estudo possibilitou expandir os conhecimentos acerca da atuação dos profissionais fisioterapeutas frente a pacientes que requeiram cuidados paliativos. Dessa forma, considera-se que novas pesquisas devem ser realizadas nesta linha de investigação, a fim de provar a importância desses profissionais e a inserção de uma equipe multidisciplinar para uma melhor qualidade de vida para os clientes que se encontram em estágios avançados ou em fase terminal. 


\section{Referências}

Azevedo, C. P. (2015). Cuidados paliativos: tratamento fisioterapêutico em pacientes com câncer (Trabalho de conclusão de curso). Centro Universitário de Brasília - UniCEUB, Brasília, Brasil.

Batista, L. S., \& Guarnieri, M. P. (2019). A importância do atendimento fisioterápico humanizado no paciente oncológico: uma revisão literária. 17º Congresso de Iniciação Científica da FASB, Barreiras, Ba, Brasil, 17.

Baldini, D. S., Salles, M. T., \& Santana, S. M. (2010). A atuação do fisioterapeuta no controle da dor oncológica: uma revisão de literatura (Trabalho de conclusão de curso). Escola Bahiana de Medicina e Saúde Pública, Salvador, BA, Brasil.

Bernardo, E. S. (2012). Pacientes oncológicos em cuidados paliativos: correlação entre qualidade de vida e funcionalidade (Trabalho de conclusão de curso). Universidade do Extremo Sul Catarinense - UNESC, Criciúma, SC, Brasil.

Burgos, D. B. L. (2017). Fisioterapia Paliativa Aplicada ao Paciente Oncológico Terminal. Ensaios Cienc., Cienc. Biol. Agrar. Saúde, $21(2), 117-122$.

Carvalho, L. O. R., Duarte, F. R., Menezes, A. H. N., \& Souza, T. E. S. (2019). Metodologia científica: teoria e aplicação na educação a distância.: PetrolinaPE: Fundação Universidade Federal do Vale do São Francisco.

Cazzaniga, M., \& Bonanni, B. (2018). Relationship Between Metabolic Disorders and Breast Cancer Incidence and Outcomes. Is There a Preventive and Therapeutic Role for Berberin?. Anticancer Research, 38 (8), 4393-4402.

Costa, E. O. (2017). Cuidados paliativos ao paciente com câncer: a atuação de fisioterapeutas n o âmbito hospitalar (Dissertação de mestrado). Universidade Federal da Paraíba, João Pessoa, PB, Brasil.

Cunha, C. V., \& Gardenghi, G. (2019). A fisioterapia nos cuidados paliativos a pacientes com câncer - uma revisão baseada em evidências (Trabalho de conclusão de curso). Instituição de Ensino Superior, pioneira no Centro-Oeste (CEAFI), MG, Brasil.

Duarte, B. C. B. (2018). Atuação do fisioterapeuta em pacientes oncológicos em cuidados paliativos em um hospital filantrópico da cidade de Maceió (Trabalho de conclusão de curso). Centro Universitário CESMAC, Maceió, AL, Brasil.

Freitas, G. S. S., Gonçalves, C., \& Morais, M.I.M. (2016). A contribuição da fisioterapia nos cuidados paliativos em crianças com leucemia, Revista UNIABEU Belford Roxo, 9(21), 182-192.

Góes, G. S., Munduruca,T. L. L., Ferreira, V., \& Passos, E. C. (2016). Atuação do fisioterapeuta nos cuidados paliativos em pacientes oncológicos adultos hospitalizados: Revisão de literatura (Trabalho de conclusão de curso). Escola Bahiana De Medicina E Saúde Pública. Salvador, BA, Brasil.

Instituto Nacional de Câncer (2019). ABC do câncer: abordagens básicas para o controle do câncer: INCA.

Marconi, M. A., \& Lakatos, E. M. (2003). Fundamentos de metodologia científica. (5a ed.). Atlas.

Matheus, L. B. G., Silva, L. L. S., \& Figueiredo, L. C. (2018). Abordagem fisioterapêutica no paciente oncológico (pp. 733-741). Diretrizes oncológicas.

Müller, A. M., Scortegagna, S., \& Moussalle, L. D. (2010). Paciente Oncológico em Fase Terminal: Percepção e Abordagem do Fisioterapeuta. Revista Brasileira de Cancerologia, 57(2), 207-215.

Nascimento, I. M. B., Marinho, C. L. F., \& Costa, R. O. (2017). A contribuição da fisioterapia nos cuidados em pacientes com dor oncológica. Rev. UNINGÁ, $54(1), 1-7$.

Oliveira, T., Bombarda, T. B., \& Moriguchi, C. S. (2019). Fisioterapia em cuidados paliativos no contexto da atenção primária à saúde: ensaio teórico. Cad. Saúde Colet., 27 (4), 427-431.

Silva, R. M. F. (2014). Recursos fisioterapêuticos no tratamento da dor oncológica (Trabalho de conclusão de curso). Pontifícia Universidade Católica de Goiás, Goiânia, GO, Brasil.

Sousa, L. M. M., Marques-Vieira, C. M. A., Severino, S. S. P., \& Antunes, A.V. (2017). A metodologia de revisão integrativa da literatura em enfermagem. Revista de Investigação de Enfermagem, 17-26.

World Health Organization (WHO). Câncer. Geneva: WHO, 2018. https://www.who.int/news-room/fact-sheets/detail/cancer 